\title{
Multidisciplinary care of a paediatric patient with Gradenigo's syndrome
}

\author{
Noor Janjua, ${ }_{1}^{1}$ Mohammed Bajalan, ${ }^{2}$ Samantha Potter, ${ }^{3}$ Andrea Whitney, ${ }^{2}$ Fabian Sipaul ${ }^{2}$
}

${ }^{1}$ Department of Trauma and Orthopaedics/ENT, Queen Alexandra Hospital, Portsmouth, UK

${ }^{2}$ Department of ENT, University Hospital Southampton

Southampton, UK

${ }^{3}$ Department of Paediatrics,

Queen Alexandra Hospital,

Portsmouth, UK

\section{Correspondence to}

Noor Janjua,

nj238cam@gmail.com

Accepted 11 February 2016

\section{CrossMark}

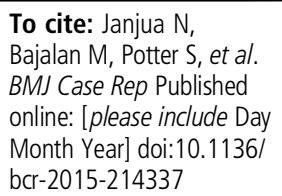

\section{SUMMARY}

A 10-year-old girl presented with signs and symptoms suggestive of Gradenigo's syndrome, a condition characterised by otorrhoea, diplopia due to abducens nerve palsy and pain in the region of the trigeminal nerve. This case examines the presentation of this condition, and the appropriate investigations. We also highlight the importance of the involvement of multiple specialities in discussing and devising a suitable management plan.

\section{BACKGROUND}

The syndrome was first described in 1907 by Guiseppe Gradenigo, a prominent Italian otologist. It is a now-rare complication of acute otitis media (AOM), consisting of otorrhoea, diplopia secondary to abducens nerve palsy and pain in the region of the trigeminal nerve. These neural effects are due to petrous apicitis causing inflammation of the nerves in Dorello's canal and Meckel's cave, respectively. Its relative rarity is primarily due to increase utilisation of antibiotics.

Case reports of Gradenigo's syndrome can be found in the literature, including recent descriptions of cases managed medically, avoiding aggressive surgical intervention. ${ }^{1-3}$ These reports, however, fail to stress the need for multiple specialty input, which is essential for the care of these patients. The need for this is irrespective of whether the condition is treated surgically or medically, as management decisions should be made only after multidisciplinary discussion.

It is common practice for all paediatric patients with periorbital cellulitis to be admitted under the joint care of the paediatric, ophthalmology and ear, nose and throat (ENT) teams.

Our case report demonstrates the necessity of a similar multiple specialty approach to decisionmaking and the subsequent influence on management.

\section{CASE PRESENTATION}

A 10-year-old girl presented to her local hospital following 2 weeks of worsening right-sided otalgia with otorrhoea and frontal headache. Within this time, she had shown some improvement to a course of oral erythromycin, before becoming increasingly unwell with lethargy, fevers, worsening headache and decreased levels of consciousness (GCS 14/15).

In the past 18 months, she had experienced an episode of reactive arthritis of the left ankle, but had no other relevant medical or family history.
She was given 3 days of intravenous ceftriaxone under the care of the local paediatric team, but showed no improvement. Subsequent transfer to the local ENT tertiary paediatric hospital for specialist input was made.

On examination, she appeared listless and sleepy, with a temperature of $39.3^{\circ} \mathrm{C}$. Her other observations included a heart rate of 77 , respiratory rate of 32 , oxygen saturations of $99 \%$ on room air and blood pressure of 116/76.

Otoscopy revealed copious amounts of purulent yellow-green discharge from the right ear, limiting examination of the canal and tympanic membrane. Positive clinical signs included a right abducens nerve palsy and hypoaesthesia in the region of the trigeminal nerve, including paraesthesia of the anterior part of her tongue. It was based on this combination of findings that the diagnosis of Gradenigo's syndrome was made.

\section{INVESTIGATIONS}

At the referring hospital, blood tests had shown mildly raised white cell count (WCC) and C reactive protein (CRP) of 13.6 and 14, respectively. Renal function, liver function and clotting were all unremarkable. CT had been performed prior to transfer, which confirmed inflammation of the right petrous temporal bone apex, so called petrous apicitis (figure 1). However, the mastoid air cells were only partially opacified. Blood tests after transfer showed that the CRP had risen to 140 , although WCC was 12.2.

Following initial transfer to the ENT team, several specialities were also involved, and included:

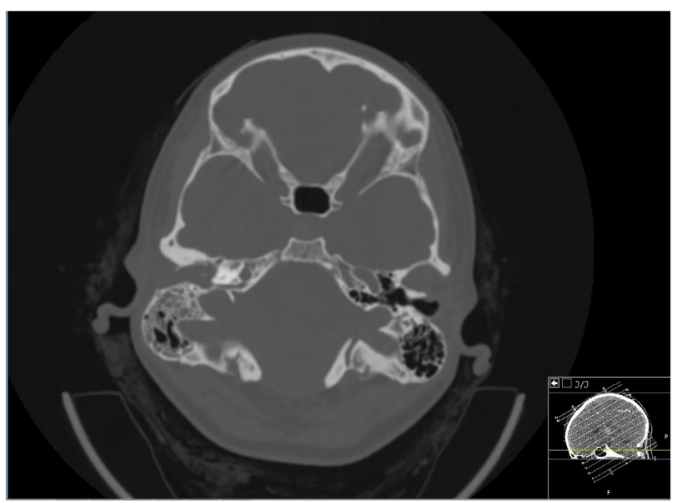

Figure 1 Axial slice of initial CT scan showing opacification of the right mastoid air cells, and high signal in the right petrous apex, consistent with petrous apicitis. 


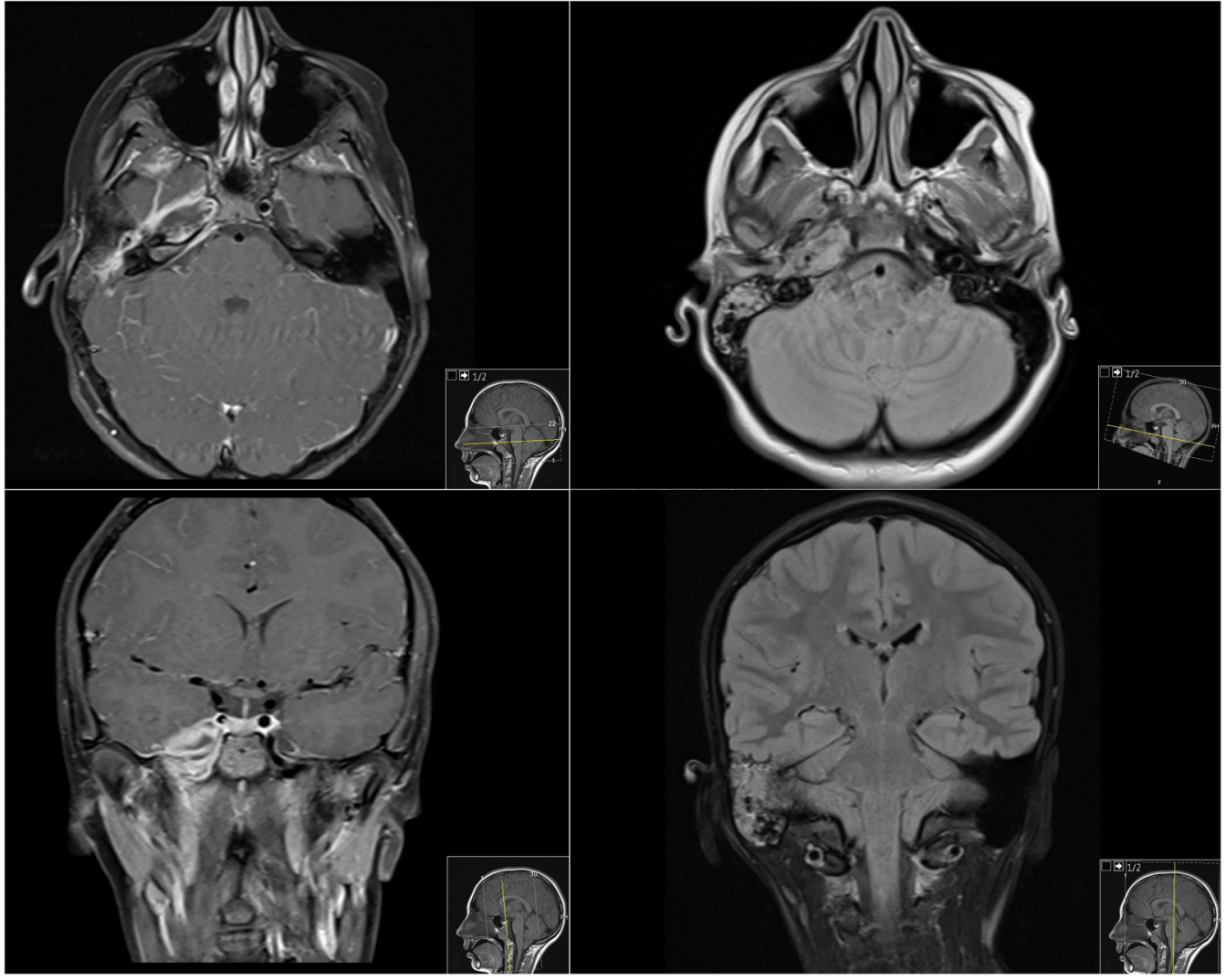

Figure 2 Axial and coronal views of MRI scan performed on transfer to tertiary centre. These show high signal in the right petromastoid complex air cells, consistent with confluent apical petrositis, and enhancing phlegmonous tissue expanding the petrous apex.

- Radiology: emergency MRI/MR venography (MRV) discussed and agreed on (figure 2).

- Microbiology: advised intravenous ceftriaxone until swab results of the ear discharge became available.

- Ophthalmology: undertook urgent assessment and identified decreased acuity of the right eye laterally within the field of view, raising suspicion of early optic nerve compromise. There was also a mild decrease in red desaturation and decreased corneal sensation. They agreed with the plan for MRI/MRV, and added the suggestion of an eye patch to counteract the disorientating effects of the diplopia.

- Paediatric neurology: urgent assessment found no other neurological deficits. They agreed with the plan for MRI/ MRV.

MRI concluded right confluent apical petrositis with an adjacent epidural abscess and right internal carotid arteritis (figure 3), as described, with no cavernous sinus thrombosis.
The report also noted avidly enhancing thickening of the meninges extending into the right internal auditory meatus, Meckel's cave and in the region of Dorello's canal (figure 2).

Following the initial MRI report, the neurosurgical team was informed, which advised no immediate surgical treatment, but recommended follow-up imaging a few days later.

The paediatric infectious diseases team also reviewed the patient and suggested a minimum 2 week course of intravenous ceftriaxone would be needed, and organised the insertion of a peripherally inserted central catheter line while adding oral metronidazole to the antibiotic plan.

\section{DIFFERENTIAL DIAGNOSIS}

The triad of signs following the history of AOM pointed towards Gradenigo's syndrome. However, given these signs, other serious complications of AOM had to be investigated and excluded, including intracranial abscesses and sinus thromboses.
Figure $3 \mathrm{MRI}$ angiography following presentation and on follow-up, showing narrowing of the petrous segment of the right internal carotid artery in keeping with right internal carotid arteritis, and subsequent resolution.

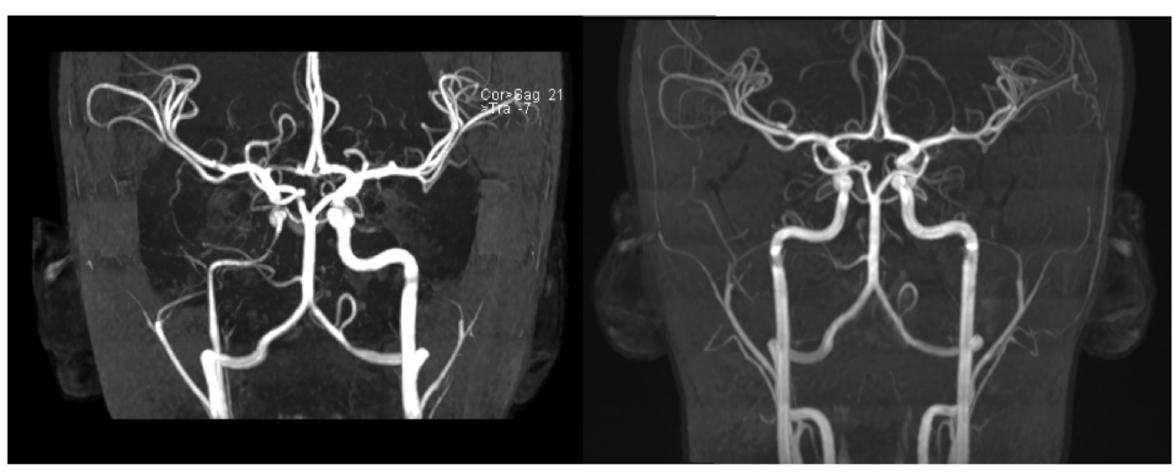

Janjua N, et al. BMJ Case Rep 2016. doi:10.1136/bcr-2015-214337 
Lateral sinus thrombosis is a well-known intracranial complication of AOM. Clinical features vary according to the stage of the disease with headache, fever and otorrhoea. Occlusion of the lumen of the sinus and interruption of the cortical venous circulation results in headache, papilloedema and increased intracranial pressure. Involvement of the sagittal sinus can result in otitic hydrocephalus. Tenderness and oedema over the mastoid (the Griesinger sign) are highly suggestive of lateral sinus thrombosis and reflex thrombosis of the mastoid emissary vein, absent in our patient's clinical examination.

As the mastoid is contiguous to and an extension of the middle ear cleft, virtually every patient with AOM or chronic middle ear inflammatory disease has a degree of mastoiditis. In most cases, middle ear symptoms predominate with fever, otalgia and conductive hearing loss. In some patients, the infection spreads beyond the mucosa of the middle ear cleft, and patients develop osteitis within the mastoid air-cell system or periosteitis of the mastoid process, either directly by bone erosion through the cortex or indirectly via the emissary vein of the mastoid. As a result, clinical signs include loss of post auricular skin crease, mastoid tenderness and erythaema and protrusion of the ear, none of which were present in our patient.

Symptoms of meningitis were present in this patient, with neck stiffness and photophobia. More general, overlapping symptoms of severe infection were also present, including fever, headache, sleepiness, confusion and irritability. She had meningeal involvement of the infection and likely had meningitis in addition but was too unwell for LP, and treated presumptively.

Intracranial abscesses are uncommon, serious, life-threatening infections. They include brain abscess, subdural or extradural empyema and are classified according to the anatomical location or the aetiological agent. They can originate from infections of contiguous structures, AOM, dental infection, mastoiditis and meningitis. Symptoms can be present for 2 weeks or less with a variable course. Most symptoms result from the size and location of the space-occupying collection. The triad of fever, headache (often severe and on the side of the abscess) and focal neurological deficit, occurs. Other clinical signs such as mental status changes, indicating cerebral oedema, focal seizures, nausea and vomiting, nuchal rigidity and papilloedema, were all absent in our patient's presentation. MRI scanning further excluded these diagnoses from our initial list of differentials.

\section{TREATMENT}

Initially, grommet insertion was performed to allow unimpeded egress of mucopus from the middle ear. This was inserted through a fresh anteroinferior myringotomy. Cortical mastoidectomy was deemed unnecessary at this stage as there was no significant opacification of mastoid air cells. Sofradex eardrops were also started.

In light of the MRI finding of internal carotid arteritis, with significant carotid narrowing within the petrous bone, the paediatric neurology team started low dose aspirin as antiplatelet therapy.

Over the next few days, the patient improved clinically, with reduced otalgia and otorrhoea. However, the diplopia and headaches remained a persistent problem. Dexamethasone therapy was started as an anti-inflammatory and subsequent analgaesic, to good effect.

After 3 days, two paediatric ENT consultants discussed the case together with a paediatric neurology and a neurosurgical consultant. They decided to plan for drainage of the extradural collection with a cortical mastoidectomy. Before this, however, an MRI was arranged to reassess the situation.

This follow-up MRI report conclusion noted 'persistent right confluent apical petrositis with decrease in the adjacent epidural abscess, meningeal enhancement and improvement of the right petrous segment internal carotid artery calibre... No cavernous sinus thrombosis...'.

Based on these findings, all the involved teams agreed to hold off any surgical intervention and continue with the medical management. Two swabs sent for microbiology grew Streptococcus intermedius, sensitive to penicillin (including ampicillin and amoxicillin), erythromycin, chloramphenicol and cotrimoxazole. One of these swabs suggested resistance to rifampicin. Ceftriaxone was deemed a suitable and practical option, given the cephalosporins' equivalent action to penicillins and ceftriaxone's once-daily dosing regime.

\section{OUTCOME AND FOLLOW-UP}

Clinical improvement continued with full abduction of the right eye, alongside resolution of the tongue paraesthesia and pain. Oral intake and mobility improved, and plans were made for discharge, with continued outpatient intravenous antibiotics by the community ambulatory team. CRP prior to discharge was 17 . The paediatric neurology and infectious diseases teams concurred on recommending a total of 6 weeks antibiotic therapy, including the time spent in hospital. The paediatric neurology team also recommended that the aspirin be continued for 3 months.

Before discharge, another MRI/MRA scan was performed. This showed a 'further improvement in the calibre of the right cavernous internal carotid artery...' The MRI report concluded, 'no progression of inflammatory change/infection over the past week...' (figures 3 and 4).

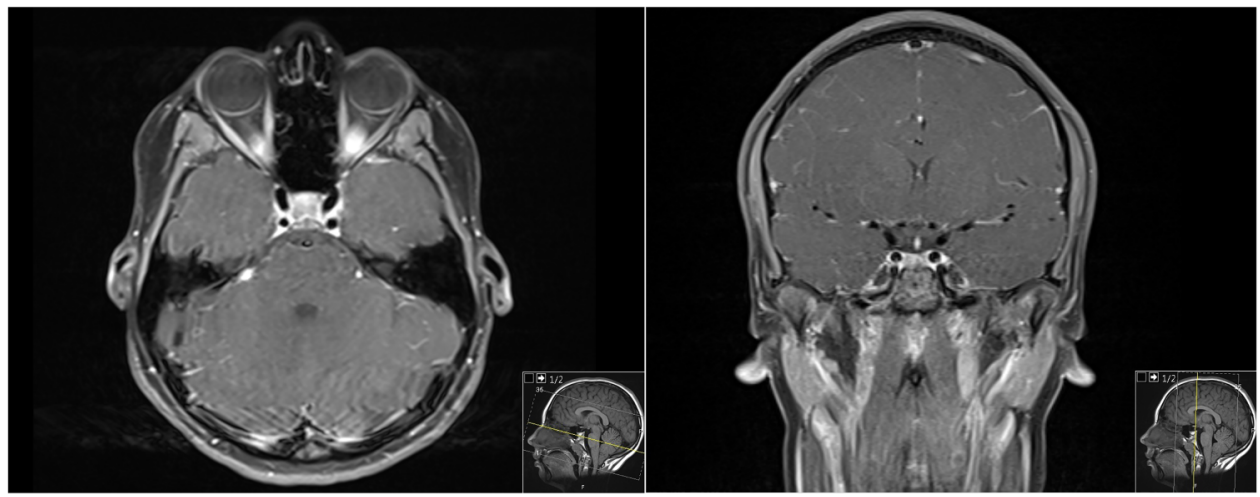

Figure 4 Axial and coronal views of most recent follow-up MRI scan, clearing of fluid signal from the right mastoid air cells and persistent but reduced signal around the right petrous apex with surrounding fatty change. 
Follow-up was arranged with ENT and paediatric neurology. At 2 months there had been complete resolution of symptoms, including neurological and this was maintained at 4 months. The grommet was still in place at both of these attendances, and the tympanic membrane appeared otherwise normal. Pure tone audiometry was also normal at 4 months. As clinical symptoms, objective examination findings and investigations had all normalised, it was felt between the specialities involved, that the previously recommended duration of treatment had been adequate.

\section{DISCUSSION}

Gradenigo's syndrome is a rare, but recognised, complication of AOM. Petrous apicitis in the acute setting is thought to arise from abscess formation in a well pneumatised petrous apex. This appears on CT as an expansile lesion within a wellpneumatised petrous apex that may have irregular margins. On MRI, this will be low intensity on T1-weighted images, high intensity on T2. Gadolinium enhancement may depict a rim of hyperintensity. ${ }^{4}$ Imaging has a vital role in diagnosis and management monitoring.

Pathophysiology of the abducens nerve palsy is due to contact of this nerve with the apex of the petrous part of the temporal bone as its runs under the petroclinoid ligament and through Dorello's canal. ${ }^{5}$ The trigeminal nerve and ganglion also lie against the petrous apex. Irritation of these nerves by inflammation of the underlying bone results in the abducens nerve palsy and trigeminal neuralgia which characterise Gradenigo's syndrome.

Other rare but recognised complications of AOM include extracranial complications such as mastoiditis (which may be further complicated by subperiosteal abscess), facial nerve palsy and labrynthitis. Intracranial complications include meningitis, extradural abscess, cerebral abscess, subdural empyema and sigmoid sinus thrombosis. These, along with Gradenigo's syndrome, are much less common in the modern medical age, following the advent of antibiotics. A thorough history and detailed clinical examination including full neurological, cranial nerve, cerebellar and head and neck assessment are vital in differentiating the wide array of possible complications. A sound anatomical knowledge is essential for the clinical assessment and subsequent correlation with potential pathological complications of AOM.

Given the diversity and potential extent of complications, it stands to reason that multiple medical teams will be involved in the care of these patients, especially the paediatric cases. No case reports so far seem to address the extent of cross-specialty liaison.

Formerly, radical surgery, usually involving mastoidectomy +/- petrosectomy +/- neurosurgical drainage with their own consequent possible complications such as facial nerve damage and further intracranial infections and thrombosis, were the treatment advocated for many serious complications of AOM, including Gradenigo's syndrome. ${ }^{6}$ However, as seen in this case, careful and continuous assessment of the patient, and good communication between multiple medical teams, can achieve a good outcome, with minimal invasive intervention.

Although good communication between the treating teams promotes a positive final outcome, care must also be given to communication with the patient and family. This was an understandably worrying and stressful time for the parents, and they were very pleased with the care their daughter received and the outcome of her treatment. However, they suggested that communication between them and the multiprofessional team could have been streamlined. For example, at times, several opinions and management plans were being discussed simultaneously. This is an important learning point for working within multiprofessional teams.

Multiprofessional team-working is becoming increasingly important especially with rising specialisation within the healthcare profession. ${ }^{7}$ This case demonstrates a very positive outcome as a result of the invaluable input of multiple specialties including ENT, general paediatrics, paediatric neurology, neurosurgery, microbiology, radiology and ophthalmology. However, it also highlights one of the difficulties of working in large teams, namely, maintaining communication with the patient and family, as well as within the team itself.

Multiprofessional team-working should enable a more integrated approach to patient care, with higher levels of communication and collaborative decision-making, ${ }^{7}$ and this should extend to the patient or family.

A suggestion put forward from this case is that, in such complex team situations, consideration should be given to choose one lead clinician to convey a unified plan to the patient and parents, rather than incomplete or changing information being given piecemeal.

\section{Learning points}

- Gradenigo's syndrome is a triad of otorrhoea, abducens nerve palsy and facial pain in the trigeminal region. It is significant, as the symptoms are due to neural oedema, and should raise the concern of intracranial complications secondary to otitis media or mastoiditis.

- Despite radiological appearances, petrous apicitis has the possibility of being managed without aggressive surgical interventions, thus avoiding their potential complications.

- Patients with Gradenigo's syndrome should be admitted under joint care of paediatricians, ear, nose and throat and neurosurgeons, with allied surgical input as necessary, with senior-led decisions. Clear and frequent communications between multiple specialities has the best chance of leading to safe and satisfactory patient outcomes.

- Dexamethasone has a role in the acute setting of this condition for, anti-inflammatory and analgaesic purposes.

- Sound anatomical knowledge is essential for the clinical assessment and subsequent correlation with potential pathological complications of acute otitis media.

Contributors $\mathrm{NJ}$ is the corresponding author and main writer of the report. MB and SP contributed to sections of the writing and in finding references. FS reviewed and edited the article, including contributing to the discussion. AW has also reviewed the article and identified images to be used.

Competing interests None declared.

Patient consent Obtained.

Provenance and peer review Not commissioned; externally peer reviewed.

\section{REFERENCES}

1 Rossor TE, Anderson YC, Steventon NB, et al. Conservative management of Gradenigo's syndrome in a child. BMJ Case Rep 2011;2011:bcr0320113978.

2 Scardapane A, Del Torto M, Nozzi M, et al. Gradenigo's syndrome with lateral venous sinus thrombosis: successful conservative treatment. Eur J Pediatr 2010;169:437-40 
3 Burston BJ, Pretorius PM, Ramsden JD. Gradenigo's syndrome: successful conservative treatment in adult and paediatric patients. J Laryngol Otol 2005;119:325-9.

4 Jackler RK, Parker DA. Radiographic differential diagnosis of petrous apex lesions. Am J Otol 1992:13:561-74.

5 Azarmina M, Azarmina H. The six syndromes of the sixth cranial nerve. J Ophthalmic Vis Res 2013:8:160-71.
6 Goldstein NA, Casselbrant ML, Bluetsone CD, et al. Intratemporal complications of acute otitis media in infants and children. Otolaryngol Head Neck Surg 1998;119:444-54.

7 Nancarrow SA, Enderby PM, Ariss SM, et al. The impact of enhancing the effectiveness of interdisciplinary team working: cost and outcomes. National Institute for Health Research Service Delivery and Organisation Programme, 2012.

Copyright 2016 BMJ Publishing Group. All rights reserved. For permission to reuse any of this content visit http://group.bmj.com/group/rights-licensing/permissions.

BMJ Case Report Fellows may re-use this article for personal use and teaching without any further permission.

Become a Fellow of BMJ Case Reports today and you can:

- Submit as many cases as you like

- Enjoy fast sympathetic peer review and rapid publication of accepted articles

- Access all the published articles

- Re-use any of the published material for personal use and teaching without further permission

For information on Institutional Fellowships contact consortiasales@bmjgroup.com

Visit casereports.bmj.com for more articles like this and to become a Fellow 